\title{
THE NSW MEN'S HEALTH INFORMATION AND RESOURCE CENTRE
}

\author{
David Crawford, Anthony Brown, and Dennis \\ McDermott \\ Men's Health Information and Resource Centre \\ University of Western Sydney, Richmond
}

\section{BACKGROUND}

The Men's Health Information and Resource Centre (MHIRC) aims to support the development of ways to enable boys and men to lead fulfilling lives and thereby contribute to a better quality of life for all members of society. A priority of MHIRC is to promote the health and wellbeing of those men and boys who are disadvantaged, including indigenous men, homeless men, and older men. To meet its aims and purpose, the MHIRC's activities focus on the following key result areas: networking and resource-information brokerage; research projects; special projects; and partnerships.

The MHIRC grew out of a partnership that developed in the mid 1990s in the Hawkesbury area between the Wentworth Area Health Service, the Hawkesbury District Health Service, the local Division of General Practice, the University of Western Sydney, and the RAAF Base at Richmond.

In August 1999, the NSW Minister for Health, Craig Knowles, released a policy document Moving Forward in Men's Health, ${ }^{1}$ which provides statewide leadership in men's health. The document recommended the establishment of a men's health information and resource centre, as 'a multi-purpose centre with functions to include research, training and information dissemination' through a range of activities, networks, publications, and projects. ${ }^{1}$

The NSW Department of Health provided recurrent funding for the establishment of a project team for MHIRC. The University of Western Sydney provides facilities at the Richmond campus, as well as two academic staffProfessor John Macdonald and Mr Michael Woods-who also act as the co-directors of the MHIRC in addition to their teaching and other duties.

\section{INITIATIVES OF THE MEN'S HEALTH INFORMATION AND RESOURCE CENTRE}

The work of the MHIRC overlaps the four focus areas, and includes the following initiatives.

\section{Networking and The Good Oil Bulletin}

Through presentations, lectures, conference papers, a web site, the Men's Health Representatives Network (described in the following article by Sliwka), and individual enquiries, MHIRC facilitates the dissemination of information about male health in NSW and beyond. In particular, The Good Oil is a free e-bulletin prepared by the MHIRC about aspects of men and boys' health and wellbeing. The Good Oil contains updates of MHIRC articles, publications, and events; as well as news about current research, programs, or items of interest in the area of men and boys' health. The bulletin is accessible at the MHRIC Web site www.menshealth.uws.edu.au.

\section{Research projects}

The MHIRC is coordinating a study that looks at men's perceptions of their health needs, and how the health system responds to those needs, across two area health services of NSW. The study, Engaging Men in the Health System, will investigate the barriers that keep many men from using existing health services, and the informal practices men are already pursuing to foster good health outcomes. At the same time, the MHIRC is piloting a longitudinal study of the health of Australian men.

As well as auspicing the research of others, and developing a new research agenda around men's health (see the Publication page at the MHIRC Web site), MHIRC brokers research into issues concerning male health. The MHIRC is collaborating with Centacare, Broken Bay-the originators of the Hey Dad! parenting program - in a project called Fathering in Diverse Communities, which looks at aspects of fathering among a range of culturally and linguistically diverse communities.

\section{Special projects in indigenous health}

The MHIRC's full-time and seconded indigenous staff have assisted with policy and program development around indigenous male health in NSW. A further initiative, being developed in partnership with local Koori men and the Holy Family Centre in Mt. Druitt, is for a Koori men's and boys' centre.

Second National Indigenous Male Health Convention Building Spirit: Building Health, the Second National Indigenous Male Health Convention, was held on the University of Western Sydney's Hawkesbury Campus in September 2001. The Convention received substantial support from MHIRC. The national coordinator was based at the MHIRC office, the MHIRC resourced the planning committee, and MHIRC staff were critically involved in organising the Convention. The two-day Convention gave delegates the opportunity to draw on the strengths of Aboriginal and Torres Strait Islander culture to combine traditional knowledge with innovative methods of health service delivery. New approaches examined include 'Rekindling the Spirit', a program from the north coast of NSW. This program has received awards for pioneering work with indigenous school age boys' and men released from custody, based on the Aboriginal concept of 'men's business'.

\section{Fourth National Men and Boys' Health Conference} The MHRIC hosted the Fourth National Men and Boys' Health Conference at the Hawkesbury campus of the University of Western Sydney, immediately following the Indigenous Male Health Convention. Approximately 300 attendees came from all parts of Australia. The aim of the Conference was to support programs and research that build men and boy's health. The Conference theme was 
'mutual respect and support', which was in evidence throughout. The format included leading speakers on men and boy's health and wellbeing - such as Richard Fletcher and Professor Faith Trent-as well as presentations, panels, and workshops covering a diverse range of men's health issues. These included physical, mental, and emotional health; men and workplace issues; health of socially disadvantaged groups of men such as indigenous, rural, and homeless men; fathers and parenting; and men's use of health services.

By adopting a social perspective of health, the conference highlighted the diversity of issues and factors that contribute to men and boy's health and wellbeing.

\section{Older men and health}

In partnership with Older Men: New Ideas (OMNI)-a social group for older men-the MHIRC conducted a number of research focus groups where older men were encouraged to discuss their perceptions of health and wellbeing. Older men are a group of potentially marginalised men who are significant users of health services. These older men saw good health as encompassing more than physical wellbeing. Health was seen as a combination and an interaction of different aspects of health, including physical, mental, emotional, 'economic health', and spiritual health. The results of this research are incorporated into a discussion paper written by the MHIRC for the NSW Committee on the Ageing, Keeping the Balance-Older Men and Healthy Ageing.
The discussion paper is being used as a starting point for reflection and action by state health agencies and community groups. Copies of the discussion paper may be obtained from the NSW Committee on the Ageing by telephone (02) 93676860.

\section{Fostering developments in men's health}

The MHIRC has been active in helping to establish the Confederation of Men's Organisations (COMO). This confederation aims to provide a forum for people working with men and boys - a place to exchange views and keep up to date with the latest information and developments in the area of men's health and wellbeing. COMO also seeks to empower men and boys and their organisations to become better advocates of men and boys' health and wellbeing.

\section{THE FUTURE}

In the eighteen months since the establishment of MHIRC as a dedicated Centre for men's health, the range of activities is constantly growing - networks, publications, and research projects-as MHIRC pursues its goals. MHIRC is evolving to meet identified needs, and is already contributing to the improvement of men's and boy's health in NSW.

\section{REFERENCE}

1. Policy Division. Moving Forward in Men's Health. Sydney: NSW Department of Health, 1999.

\section{MEN'S USE OF GENERAL PRACTITIONER SERVICES}

\author{
Michael Woods \\ Men's Health Information and Resource Centre \\ University of Western Sydney
}

The use, or rather the non-use, of health services by men is currently one of the main concerns in men's health. The Health of the People of NSW-Report of the Chief Health Officer, 2000 notes that men access health services (that is, hospital and general practitioner services as well as other providers such as naturopaths and telephone counselling services) at a lower rate than females. ${ }^{1}$ It also notes that men use preventive health services at a lower rate than women (although there are fewer preventative and screening services directed at men). ${ }^{1}$ Given that men show a higher level of serious morbidity, and have a lower life expectancy in all age groups, this comparatively low usage of services is surprising. Men's use of the major form of primary health care, general practitioners, is estimated to be at least 15 per cent lower than that for women. For example, a recent Australian study shows that men use general practitioner services on 42 per cent of all occasions of service. ${ }^{2}$ This article examines possible explanations that emerge from the literature for this pattern of usage, and describes the findings of a recent study of general practitioners (GPs) undertaken in Sydney.

The literature offers two main types of explanation to account for this lower usage rate of GP services by men, and these explanations are likely to be relevant to considering questions of men's use of other health services. The first focuses on how culture influences individual behaviour. This explanation suggests that our culture conveys different values regarding health to each gender, and that men have not been encouraged to place the same premium on health that women do. ${ }^{3,4}$ For example, a study by Jones of a sample of men in rural Queensland indicated that health only became a priority for men once it is under threat from illness or injury. ${ }^{5}$ These men equated health as 'being able to work'. This relative undervaluing of health by men in Australia can also be seen to be reflected at the level of health policy, planning and provision, in the lack 